\title{
Portafolio estudiantil, estrategias innovadoras para el aprendizaje del tema de respiración celular
}

Dilcia Sánchez

dilcia.sanchez@unah.edu.hn

Escuela de Biología

Universidad Nacional Autónoma de Honduras

\section{Resumen}

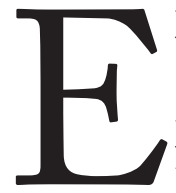

1 tema de la respiración celular forma parte de la asignatura Biología Médica, que ofrece la Escuela de Biología a los estudiantes de la carrera de Medicina de la Universidad Nacional Autónoma de Honduras, UNAH. Los datos de rendimiento académico en el aprendizaje de este tema reflejan la necesidad de mejorar el nivel de logro de los objetivos educativos.

Es así como surge el proyecto Portafolio estudiantil, estrategias innovadoras para el aprendizaje del tema de respiración celular, innovación educativa que se implementó en el tercer periodo académico 2015 y el primer período académico 2016.

El objetivo principal del proyecto fue crear los instrumentos didácticos para la implementación de una metodología innovadora para el aprendizaje del tema de respiración celular, basada en competencias.

En el presente artículo se comparte el proceso llevado a cabo, los resultados de esta experiencia de innovación docente y estudiantil, así como los productos didácticos generados para innovar la forma de abordar este tema en la clase.

Palabras Claves: Competencias, innovación educativa, objetos de aprendizaje, portafolio, respiración celular.

\section{Abstract}

The topic of cellular respiration is part of the Medical Biology class, offered by Biology School to medical career students of the National Autonomous University of Honduras, UNAH. The academic performance data about the apprenticeship on this topic reflects the need to improve in the achievement of educational objectives.

It's like that, that the project of Student Portfolio, inno- vative strategies on cellular respiration learning arises, an educative innovation that was implemented in the third academic period 2015 and the first academic period 2016.

The main objective of the project was to create teaching tools for the implementation of an innovative methodology for learning the subject of cellular respiration, based on competences.

In this article, we share the process carried out, the results of this experience of teaching innovation, as well as the didactic products generated to innovate the way to approach this topic in the class.

\section{Key words:}

Competences, educational innovation, learning objects, portfolio, cellular respiration

\section{Introducción}

Desde el año 2009, la UNAH puso en marcha su modelo edu- 
cativo que establece los lineamientos generales curriculares, pedagógicos y didácticos que fundamentan y orientan el desarrollo integral del profesional egresado de esta Institución.

Este Modelo Educativo de la UNAH se centra en los principios de: calidad, equidad, internacionalización, interdisciplinaridad; y se desarrolla bajo las siguientes perspectivas pedagógicas: la Teoría Constructivista, la Teoría Crítica y la Teoría Humanista.

Bajo este modelo se busca construir una Universidad capaz de responder a las exigencias de innovación, creatividad y cambio; el modelo centra su atención en los estudiantes en cambio los docentes se convierten en mediadores pedagógicos; se hace énfasis en aprender a aprender, aprender a enseñar, aprender a hacer,

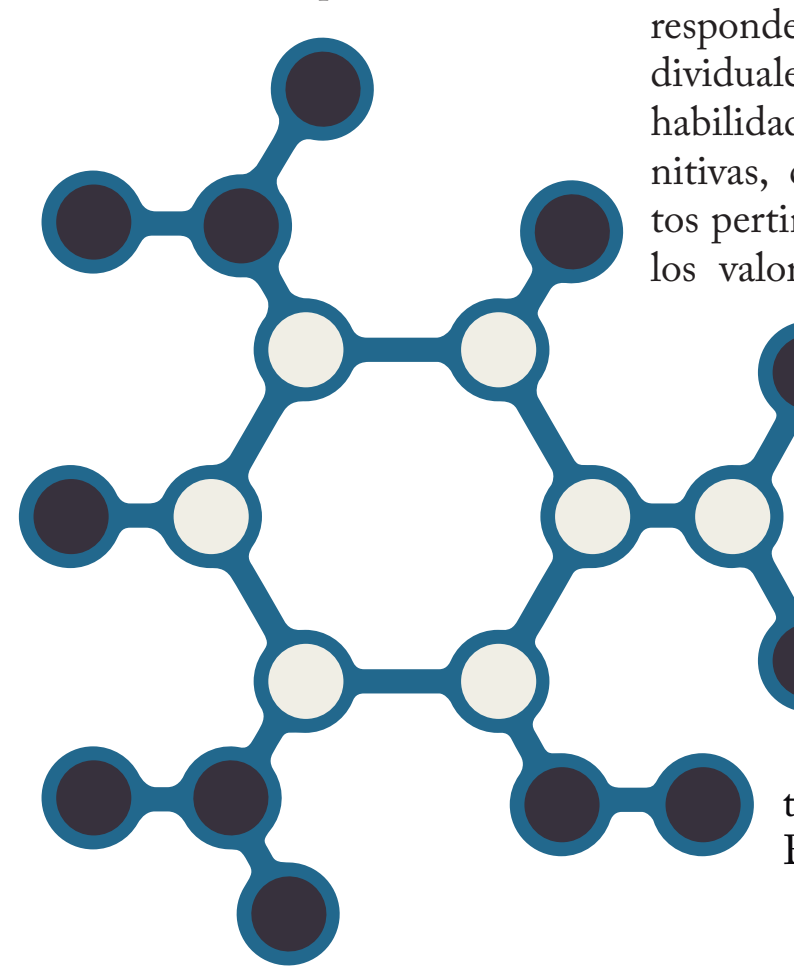

En suma, se pretende lograr ambientes de aprendizaje donde los estudiantes y futuros profesionales universitarios adquieran una formación académica que les permita responder a las exigencias inhabilidades prácticas y cognitivas, con los conocimientos pertinentes, la motivación, los valores, las actitudes, las emociones $y$ otros elementos sociales y de comportamiento que deben activarse para actuar de manera eficaz ante las demandas laborales y ciudadanas (Instituto de Tecnologías Educativas, 2010).
De ahí que uno de los modelos curriculares que establece el modelo educativo de la UNAH es el enfoque por competencias. Las competencias se definen como capacidades o habilidades para efectuar tareas o hacer frente a situaciones diversas de forma eficaz en un contexto determinado y para ello es necesario desarrollar actitudes, destrezas, conocimientos y valores de manera interrelacionada, en donde los alumnos son vistos como gestores del conocimiento. Esto les permite adaptarse a los continuos cambios que se dan en la sociedad. Bajo este enfoque los docentes son guías pedagógicos (Frola \& Velásquez, 2011).

En este contexto, para la puesta en marcha de este modelo educativo, la UNAH implementó un programa de capacitación a fin de dotar a su profesorado de las capacidades para innovar en sus prácticas docentes y hacer viable los cambios y transformaciones desde el currículo hasta el salón de clases.

Es así como se desarrolla el programa Aprender basado en el currículo TALBOK que desarrolla competencias docentes para el diseño, y gerencia de ambientes de aprendizaje efectivos (UNAH, 2008).

Varios docentes de la hoy Escuela de Biología participaron de este programa de capacitación, lograron desarrollar capacidades en el dominio de 
metodologías de enseñanza, aprendizaje y evaluación innovadoras. Esto permitió mejorar algunas estrategias educativas para el aprendizaje de temas como la Respiración Celular en la asignatura de Biología Médica; luego se tomó la decisión de llevar a cabo una intervención educativa con el proyecto "Metodologías innovadoras y portafolio estudiantil".

Es de referir que este tema registra en los estudiantes, estrés y miedo, dado que su comprensión presenta un alto grado de complejidad, según lo demuestran los resultados obtenidos en las diversas evaluaciones realizadas a los estudiantes de primer año de pregrado de la carrera de Medicina y Nutrición, de la UNAH.

La clase de Biología Médica se imparte durante cuatro horas semanales de teoría y tres horas de laboratorio, aunque no hay un laboratorio específico para el tema de Respiración Celular.

La respiración celular es un tema básico en la enseñanza de la biología ya que permite entender cómo los organismos extraen energía de las moléculas orgánicas contenidas en los alimentos a través de células especializadas (Solomon, Berg y Martin, 2011). Por ello, su comprensión y aprendizaje es vital para que los estudiantes puedan dominar otros conceptos más complejos relacionados con el tema de la salud.
El presente trabajo muestra los resultados obtenidos por los estudiantes de Medicina y Nutrición matriculados en la asignatura de Biología Medica en el III periodo del 2015 y I periodo del 2016. En las secciones de Biología Médica de estos dos periodos se puso en práctica la Unidad Didáctica del tema de la Respiración Celular basada en Competencias, se manejaron metodologías innovadoras utilizando como herramienta el portafolio estudiantil. Se trabajó con dos grupos de estudiantes, con un grupo se trabajó la metodología tradicional y con el otro la metodología innovadora.

\section{Metodología}

El presente proyecto surgió de la aplicación de principios prácticos y herramientas del currículo TALBOK en especial del diseño de Objetos de Aprendizaje, y Gestión del Conocimiento donde se forma al estudiante como futuro gestor de su propio conocimiento, equipándolo con habilidades, destrezas, actitudes y valores.

Uno de los productos del proyecto fue la elaboración de la "Unidad Didáctica con base en competencias para abordar el tema de respiración celular aplicando estrategias innovadoras para la asignatura de Biología Médica”, en la que se describen los objetivos, competencias, estrategias de enseñanza que utilizará el profesor y estrategias de aprendizaje para los alumnos.
El segundo producto del proyecto fue el diseñó una "Guía pedagógica y metodológica del docente para abordar el tema de Respiración Celular en base a competencias" en la que se detallan diversas estrategias didácticas innovadoras de enseñanza y aprendizaje que el docente puede aplicar dentro y fuera del salón de clase, entre ellas: simulaciones, juegos (crucigramas, sopa de letras), desarrollo del pensamiento crítico, aprendizaje basado en problemas, trabajo en equipo, expresión oral, elaboración de un portafolio de evidencias del estudiante, guías de estudio o cuestionarios, elaboración de resúmenes, glosarios, mapas conceptuales, esquemas, cuadro sinóptico, lotería y una actividad especial llamada Dramatización del proceso de Respiración Celular que tuvo como resultado un video del proceso. La mayoría de los estudiantes elaboraron estas actividades de aprendizaje apoyándose en el uso de las TIC para mejorar su experiencia de aprendizaje.

La programación del espacio de aprendizaje de Biología Médica para el III período académico del 2015 fue de seis secciones, con un total de 294 estudiantes, los cuales se dividieron en dos grupos para poner en práctica esta experiencia de aprendizaje:

Grupo experimental: participaron un total de 158 estudiantes a quienes se les aplicaron 
las estrategias didácticas innovadoras especificadas en la "Guía pedagógica y metodológica del docente para abordar el tema de Respiración Celular con base en competencias" acordes con el modelo educativo de la UNAH.Grupo control: Participaron un total 136 estudiantes de otras secciones en las que los docentes desarrollaron el tema de respiración celular de forma tradicional, como se ha venido enseñando en la Escuela de Biología.

La unidad didáctica para el desarrollo del tema se elaboró tomando en cuenta las competencias conceptuales, procedimentales y actitudinales. Esto les permitió a los estudiantes

del grupo experimental ser los gestores de su propio conocimiento mediante la elaboración de diversos objetos de aprendizaje haciendo uso de las TIC, especificados en una guía metodológica, siendo estos: crucigramas, sopa de letras, mapas conceptuales, glosarios, resúmenes, esquemas, lotería y presentaciones entre otros.

Asimismo, como parte de estas buenas prácticas educativas, los estudiantes elaboraron un portafolio impreso de evidencias donde registraron cada una de las actividades de aprendizaje realizadas, que serían los objetos de aprendizaje como los mencionados anteriormente. Como metodología de aprendizaje y evaluación, el portafolio estudiantil

Gráfico 1

Comparativo de resultados según la aplicación de estrategias Biología Médica. II parcial III periodo año 2015

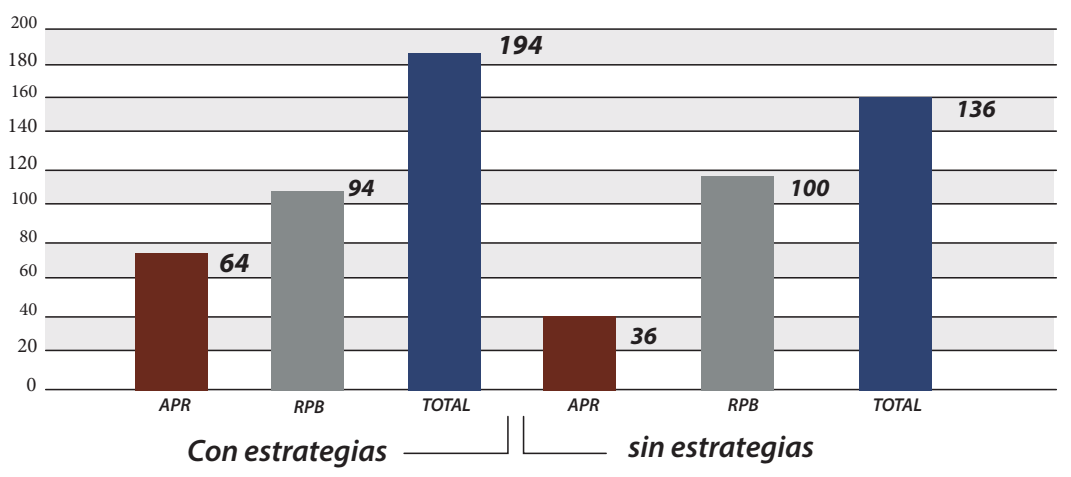

Fuente: Resultados del II parcial Respiración Celular BI-123 III periodo 2015 UNAH.

representa una fuente de información confiable, fomenta el desarrollo integral para la asimilación de los contenidos y deja las huellas de sus experiencias y reflexiones durante el proceso educativo (Manrique, 2014).

Una de las actividades de aprendizaje más significativo fue la dramatización del proceso de respiración celular, realizada por los estudiantes de manera grupal y colaborativa donde los actores fueron los mismos estudiantes, quienes se prepararon durante semanas. Esta actividad fue grabada y editada.

Además, se elaboró con el apoyo de la Dirección de Innovación Educativa un espacio virtual de apoyo a la presencialidad de la asignatura de Biología Médica, específicamente para apoyar el tema de Respiración Celular, este espacio contiene las instrucciones de cada una de las actividades de aprendizaje que el estudiante debe realizar durante el periodo. En este espacio se colocaron en formato digital, algunos de los mejores trabajos elaborados por los estudiantes, con el fin de compartir el conocimiento con otros estudiantes.

\section{Resultados}

Al finalizar el proyecto se valoraron los aprendizajes alcanzados a través de un proceso de evaluación formativa y sumativa. Se hizo el análisis estadístico estableciendo comparaciones sobre los aprendizajes logrados por todos los participantes del grupo control y experimental.

A continuación, se muestran los resultados de la aplicación de la metodología y estrategia innovadora aplicada durante el tercer período académico del año 2015 y primer periodo 2016 en el tema Respiración Celular de la asignatura de Biología Médica de la Escuela de Biología de la UNAH

En el grupo control, participaron 136 estudiantes con quienes se desarrolló el tema 
Gráfico 4

Alumnos aprobados y reprobados en Biología Medica sin utilizar estrategias innovadoras

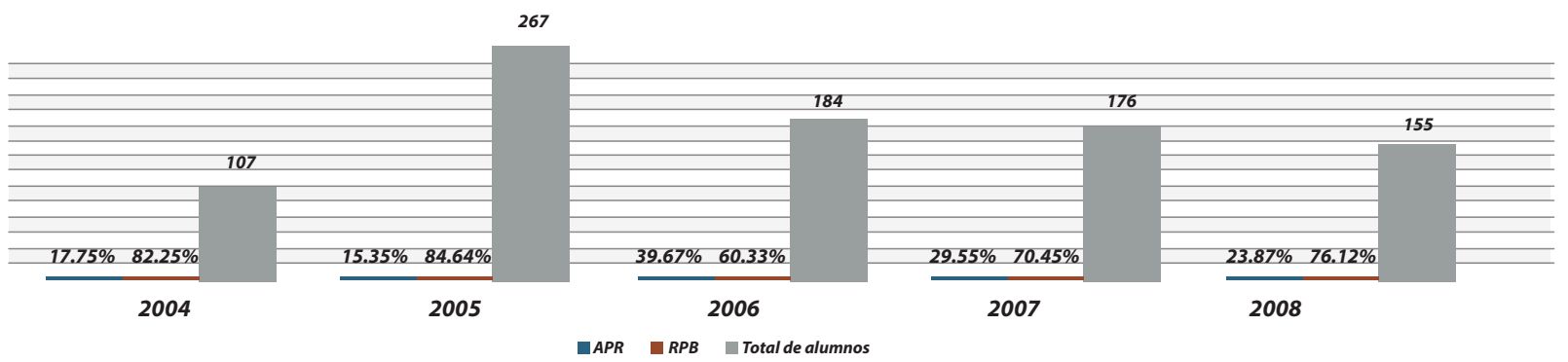

Fuente: Resultados del II parcial Respiración Celular BI-123 III periodo 2015 UNAH.

de respiración celular de forma tradicional. El total de reprobados fue de 100 alumnos equivalente al $74 \%$ y aprobados 36 que representa un 26\%, según el gráfico No.1.

En el grupo experimental, participaron 158 estudiantes. Con ellos se aplicaron diversas Estrategias Innovadoras para el desarrollo del tema de respiración celular. De los cuales reprobaron el examen 94, equivalente al 66\% del total; los alumnos que aprobaron fueron 64 quienes representan el 34\% (ver gráfico 1).

Los resultados obtenidos muestran que el porcentaje de aprobados fue significativo para los estudiantes con quienes se utilizó una metodología innovadora si se comparan con los estudiantes del grupo control que utilizaron una metodología de aprendizaje tradicional.

La metodología innovadora utilizada que incluyó la elaboración de objetos de aprendizaje y de un portafolio de aprendizaje aportó al desa-

Gráfico 2

Rango de notas del segundo parcial secciones que se aplicaron estrategia

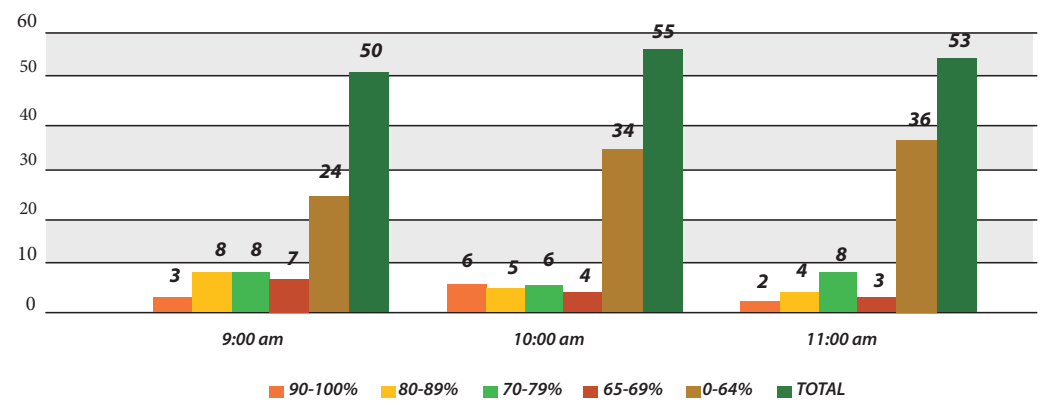

Fuente: Resultados del II parcial Respiración Celular BI-123 III periodo 2015 UNAH.

Gráfico 3

Alumnos agrupados por rango de notas secciones que no aplicaron estrategias Biología Médica II parcial, III periodo año 2015

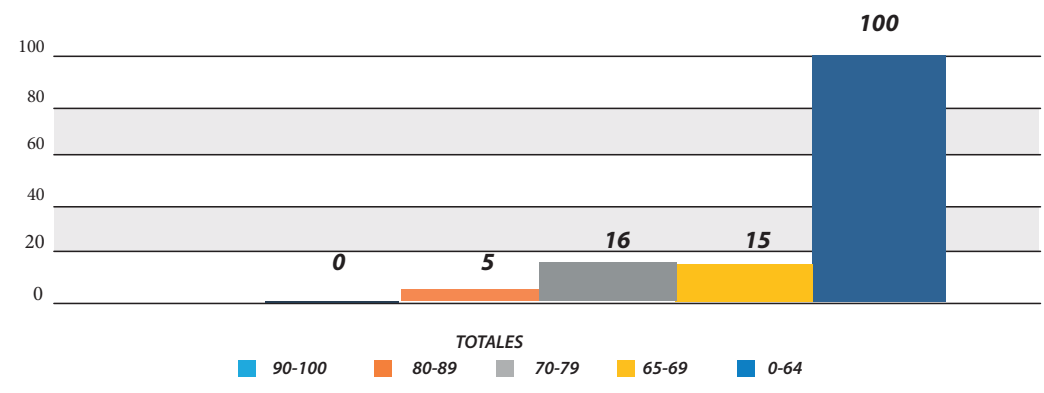

Fuente: Resultados del II parcial Respiración Celular BI-123 III periodo 2015 UNAH.

rrollo de competencias para el análisis y la síntesis de información, desarrollo del pensamiento crítico, trabajo colectivo con ética y calidad, uso de las TIC y la comprensión de las fases de la respiración celular.
Los resultados obtenidos muestran que la aplicación de las estrategias innovadoras propuestas ayudó en gran manera a la mayoría de los estudiantes a lograr los objetivos educativos y aprobar el examen parcial, lo cual se vio reflejado en su índice académico. 


\section{Gráfico 5 \\ Estudiante de biología médica aprobados y reprobados usando estrategia innovadoras I periodo del 2016}

\section{Serie $1 \quad 248$}

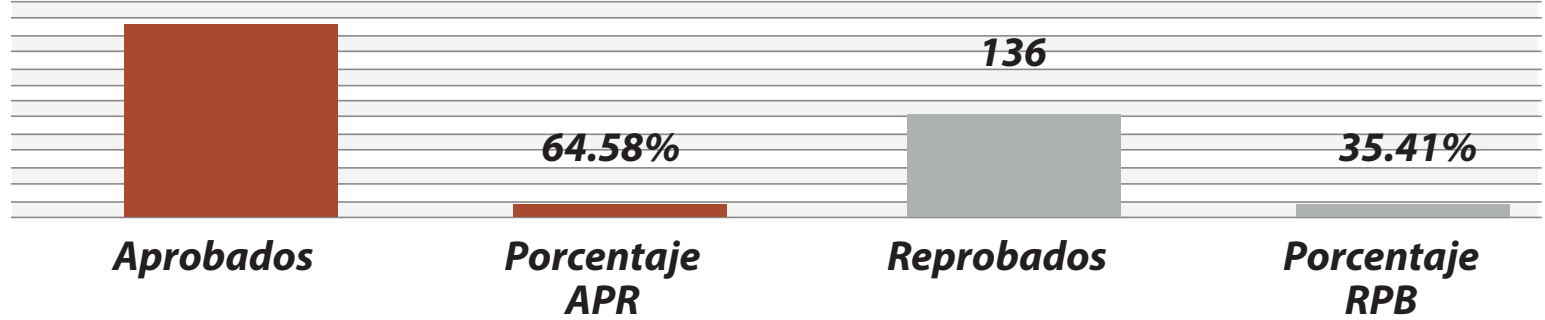

Fuente: II Examen parcial de BI - I PAC 2016 UNAH.

Las gráficas 2 y 3 muestran el rendimiento académico alcanzado por los estudiantes del grupo control y el grupo experimental en la evaluación del tema respiración celular en el segundo parcial del III Período del 2015.

Los resultados mostrados en los gráficos 2 y 3 indican que 28 de los estudiantes que elaboraron sus propios objetos de aprendizaje sobre el tema desarrollado, lograron obtener notas de excelencia académica que oscilaron entre 100\% y $80 \%$ y de ellos, 8 obtuvieron entre $100 \%$ y $90 \%$. Se evidencia el nivel de logro educativo comparado con el grupo control en el que no hubo notas entre $100 \%$ y $90 \%$, solo 5 de ellos lograron obtener notas de entre $80 \%$ y $90 \%$.

Para el logro de los objetivos educativos del tema respiración celular desarrollado de forma innovadora, los estudiantes realizaron actividades de lectura interpretativa, resumieron, investigaron, analizaron diaria- mente toda la información para luego pasar a la construcción de los objetos de aprendizaje y elaboración del portafolio estudiantil. La evaluación utilizada fue formativa y sumativa, esto permitió al estudiante observar su nivel de desempeño y obtener una retroalimentación que mostró un alto impacto en la evaluación final.

Estos resultados son significativos ya que, en años anteriores, el tema de respiración celular se impartía de forma tradicional y era casi imposible observar notas de excelencia académica en este parcial según lo muestran los datos del 2004 al 2008 presentados en el gráfico 4.

En estos años, se matricularon 889 estudiantes de los cuales, reprobaron 665 equivalente al $74.80 \%$, estos resultados son bastante altos comparados con los obtenidos durante la aplicación de las Metodologías Innovadoras. Desde esta mirada, el proyecto de innovación llevado a cabo ha sido un éxito, tanto para los estudiantes como para el docente que diseñó una nue- va propuesta didáctica para el logro de aprendizajes significativos por parte de sus estudiantes de Biología Médica.

Debido a los resultados de mejora en los aprendizajes obtenidos en este proyecto de innovación educativa aplicado en 2015, se replicó de nuevo su aplicación en el primer período del año 2016, obteniéndose resultados de mejoría de los aprendizajes similares al 2015, como se muestra en el gráfico No 5 .

\section{Conclusiones}

Los resultados de logro de objetivos educativos obtenidos en la aplicación de la propuesta didáctica innovadora en la enseñanza y aprendizaje del tema de respiración celular en el III período académico 2015 y I periodo 2016, evidencian la efectividad de las estrategias didácticas seleccionadas por el docente y utilizadas para el aprendizaje de sus estudiantes.

Se demuestra la necesidad de que el docente realice innova- 
ciones educativas para resolver problemas de aprendizaje de los alumnos, ya que, con la mejoría de los resultados obtenidos tanto a nivel formativo como sumativo en el rendimiento académico de los alumnos en la clase de Biología Médica, se logró mostrar que el problema se debía a la estrategia tradicional usada por el profesorado.

La definición de nuevas rutas de aprendizaje para los alumnos demanda que el docente aplique las capacitaciones que brinda la UNAH y pueda establecer una planificación didáctica dinámica y creativa de sus asignaturas, lo que desde luego puede significar un reto para el docente, pero que a través de este proyecto se ha demostrado que es posible y viable.

Los resultados muestran además la efectividad del uso del portafolio estudiantil como estrategia de evaluación al igual que la dinámica de aprendizaje a través de la elaboración de objetos de aprendizaje por parte de los estudiantes; además, esto crea un ambiente propicio para el trabajo colaborativo.

Lo relevante del proyecto de innovación educativa en la asignatura de Biología Médica es que la estrategia didáctica innovadora queda documentada a través de una guía metodológica disponible para otros docentes que desean aplicar estas innovaciones en otras asignaturas. Al igual los estudiantes podrán sugerir a sus docentes este tipo de innovaciones ya que no se debe olvidar que en el nuevo escenario formativo pueden surgir rutas de aprendizaje en asociación de docentes-estudiantes.

\section{Referencias}

Manrique, V. (2014). ¿Qué es un portafolio estudiantil? ogoogle.com/site/tallerdeeticaveronicamanrique/ evidencias-de-aprendizaje-por-unidad/unidad-i-elsentido-de-aprender-sobrela-etica/-que-es-un-portafolio-estudiantil

Frola, P., \& Velásquez,J. (2011). Estrategias Didácticas por Competencias. México: Centro de Investigación Educativa.

Instituto de Tecnologías Educativas. (2010). Habilidades y competencias del siglo XXI para los aprendices del nuevo milenio en los países de la OCDE. Recuperado de: http://recursostic.educacion.es/blogs/europa/ media/blogs/europa/informes/Habilidades_y_competencias_siglo21_OCDE. pdf

Universidad Nacional Autónoma de Honduras (2008). Módulos del Proyecto Aprender.

Universidad Nacional Autónoma de Honduras (2009).
El Modelo Educativo de la UNAH. Tegucigalpa, Honduras

Solomon, Berg, \& Martin. (2011). Biología. México: Mc Graw Hill. 